\title{
Measurement of the Internal Diameters of Metallic Capillary Tubes
}

\author{
By Charles T. Collett, John C. Hughes, and Francis C. Morey
}

\begin{abstract}
As a part of a cooperative research project of the National Bureau of Standards and the National Advisory Committee for Aeronautics on the viscosity of gases at elevated temperatures and pressures, it was found desirable to study the uniformity of the internal diameters of some metallic capillary tubes. This was accomplished by means of a small thread of mercury, using X-ray technique, following the method of Fisher. Eight tubes out of a group of twelve were selected as suitable for use in an absolute viscosimeter, and flow constants were computed. The tubes studied were about 15 feet long, with an internal diameter of about 0.015 inch. Maximum deviation from the average diameter in most cases was about 1.5 percent or less.
\end{abstract}

\section{Introduction}

A current cooperative research project of the National Bureau of Standards with the National Advisory Committee for Aeronautics involves the determination of the viscosity of gases at elevated temperatures and pressures, with particular reference to exhaust gases.

The method selected for the measurements is the classical capillary flow method, and in this connection the need for the determination of the internal diameters of some metallic capillary tubes arose. For use in absolute determinations, it is necessary to know not only the average internal diameter of the tube, but also the effect of irregularities of the diameters upon the flow characteristics.

Fisher ${ }^{1}$ solved this problem with glass capillaries by measuring the length of a small thread of mercury placed in the tube and moved along it by air pressure. Variations in the cross-sectional area are detected as changes in length of the mercury thread. This method is used in selecting uniform capillary tubes for high-grade thermometers. Since the mercury thread was very small, Fisher did not attempt to determine its volume by weighing, but calculated it from the weight of a much longer thread by a method that will be considered in detail later.

The viscosimeter capillaries selected are metallic tubes of internal diameters about 0.015 in., wall thicknesses 0.005 to 0.014 in., and about $15 \mathrm{ft}$ long. Manufacturers of such tubing were unable to supply any data on variations of diameter of commercial tubing except those based on experience in measurements made on the ends of the tubes. All felt confident that the variations would be of the order of $\pm 0.0005 \mathrm{in}$. or less. It was therefore decided to try to apply Fisher's method to the problem by making X-ray photographs of mercury threads in the tubing.

\section{Description of Apparatus and Method}

\section{The short-thread measurements}

The apparatus used in the X-ray inspection of the capillary tubes is shown in figures 1 and 2 . The

\footnotetext{
W. J. Fisher, Fhys, Rev. 28, 73 (1909)
}

X-ray head, $A$, was suspended from a support so as to provide a beam directed vertically downward, through a slot in a lead shield, $B$. The capillary tube was carried on a frame, $C$, arranged to slide under the shield. A clamp, $D$, and blocks, $E$, were provided to facilitate advancing the tube 1 in. between exposures.

The capillary tube may be seen in figure 2 at $F$. It was held under slight tension in the frame and taped with cellulose tape to cross supports at intervals of about $3 \mathrm{ft}$. A plate-holder, $G$, could be clamped to the lower side of the frame, holding the glass photographic plate, $H$, enclosed in black paper envelope, tightly against the frame and in contact with the capillary tube.

The mechanism used to advance the mercury thread is seen in figure 2. A plastic cylinder, $\dot{I}$,

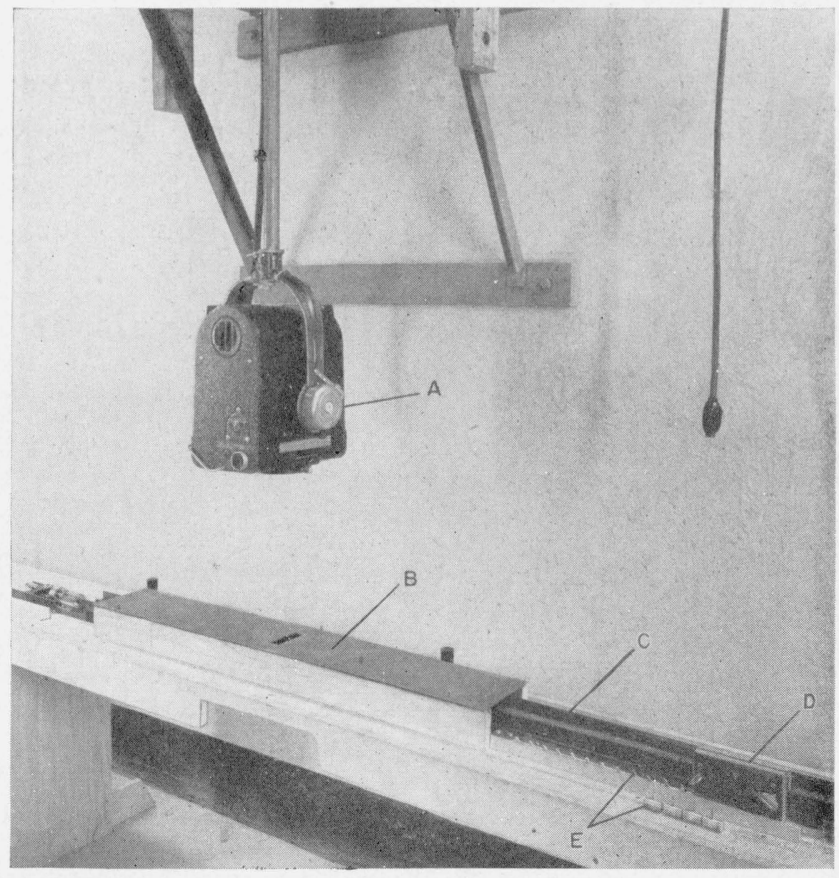

Figure 1. General view of apparatus.

$A$, X-ray source; $B$, lead shield with exposure aperture; $C$, rack for holding capillary tube; $D$, adjustable stop for positioning rack; $E$, removable blocks for positioning rack. 
was attached to the capillary tube. A plunger operated by a micrometer screw, $J$, entered the cylinder through a packing sleeve. The cylinder was filled with a petroleum solvent oil, which entered the capillary and caused the mercury thread to move along the tube. A cushion of air about $4 \mathrm{in}$. long was always maintained between the thread and the oil. The plunger diameter was selected so as to cause the thread to advance $1 \mathrm{in}$. for each five divisions on the micrometer screw.

It was not found possible to rely solely upon the settings of the micrometer screw in advancing the mercury thread, as any slight drift due to irregularities of bore, temperature changes, or other factors

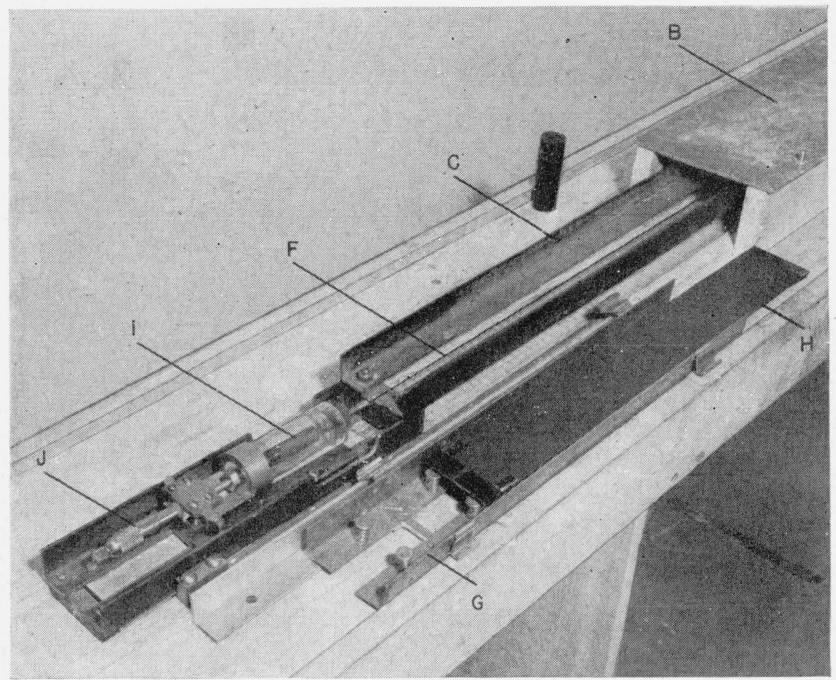

Figure 2. Creneral view of apparatus.

$B$, Lead shield; $C$, rack for holding capillary tube; $F$, capillary tube; $G$, plate holder with pressure springs; $H$, photographic plate in paper envelope; $I$, liquid cylinder for positioning mercury slug; $J$, micrometer screw for advancing plunger.

might cause the thread to travel out of the exposure field. For this reason, strips of film were placed under the supporting table, about 3 in. below the capillary and exposed simultaneously with the plate. These check films were developed in a rapid developer and examined before making the next setting. Every fourth exposure was checked in this fashion.

The photographs were made on spectrographic plates, 2 by 18 in., on each of which 17 exposures were made. An overlap of one frame was provided on successive plates, and in these frames a series of identification notches cut into the supporting framework provided a means of automatically recording the position and for carrying over the measurements from one plate to the next. All measurements were referred to a small marker, which was hard-soldered to the tube near one end.

The apparatus was located in a subbasement corridor, in which temperature variations were found to be less than $0.5^{\circ} \mathrm{C}$ during the 6 -hr period required for completing the $190 X$-ray photographs of each tube. This was found to be sufficiently uniform to make temperature corrections unnecessary.
Thread lengths ranged from approximately 0.25 to 0.40 in. Measurements of the lengths of the thread images were made with a projection microscope at a magnification of about 50 diameters. In this apparatus, the plate being measured is placed upon a mechanical stage, displacement of which can be measured with a micrometer screw reading to $0.0001 \mathrm{in}$. It was found that repeated measurements of the same photograph differed from the average value by about \pm 0.0002 in. The possibility of shrinkage of the emulsion during processing and handling was eliminated by using glass plates and avoiding use of the portions near the edges. During the first part of the work, a ball of known diameter was photographed in each frame and its image measured. When no shrinkage was observed in many photographs, its use was discontinued.

Cleaning agents were passed through the tubing with the aid of the house vacuum, and drying was accomplished by a current of air with moderate heating obtained by passing an electric current through the tube. The tube was degreased with trichloroethylene, dried; treated with concentrated nitric acid with heating nearly to boiling; rinsed with distilled water and thoroughly dried.

\section{The long-thread measurements}

As previously mentioned, it is not practical to determine the volume of the short mercury threads by direct weighing, as the mass is so small in proportion to a suitable container. It was therefore necessary to calculate this volume from the weight of a long mercury thread. The filling was accomplished by a vacuum method that was found necessary to eliminate air pockets. The tube was attached to a rotary oil-sealed vacuum pump. The opposite end was welded shut in a helium atmosphere with a tungsten electrode. The tube was then alternately evacuated and flushed with helium, while being heated electrically. This thoroughly dried the tube.

The welded closure was then removed and the open end of the tube inserted in a mercury reservoir made from a thin-walled glass tube 2 or $3 \mathrm{~mm}$ in diameter and about $15 \mathrm{~cm}$ long, which had been partially filled with mercury by vacuum distillation and had been weighed. The reservoir had a small bulb at the lower end so that air that might be carried down into the mercury by capillary action between the metal tube and the glass wall of the reservoir could not reach the end of the tube when fully immersed.

The tube extended vertically from the reservoir for a distance of about $3 \mathrm{ft}$ and then horizontally to the pump. When the pump was started, mercury entered the tube and partially filled the vertical section, acting as a barometer. Evacuation was continued for 10 to $15 \mathrm{~min}$ to insure thorough removal of the air above the mercury. The tube was then welded closed at the pump end by using the helium atmosphere and tungsten electrode. Next, the mercury reservoir was slowly raised, permitting the mercury to flow in and fill the horizontal portion of the tube. 
When the entire tube was horizontal and mercury had ceased entering the capillary tube, the tube was withdrawn from the mercury, but the end was kept within the reservoir. By gently warming the capillary tube, several drops of mercury were expelled and returned to the reservoir. Upon cooling, this left an air space several inches long near the end of the tube. The tube was then closed by welding. The mercury reservoir was again weighed. The weight of mercury entering the tube was about 5 to $7 \mathrm{~g}$, and the initial weight of the reservoir approximately $15 \mathrm{~g}$, permitting a good measurement of the weight of mercury entering the tube.

After filling, the length of the thread was determined from X-ray photographs, which were made without moving the capillary between exposures, so as to minimize the opportunity for movement of the -thread inside the tube between exposures. After the end sections had been photographed, the entire tube was X-rayed and the photographs examined under magnification of 50 diameters to detect possible voids in the thread.

\section{Method of Calculation}

The following method of calculation (except for corrections for height of meniscus) is essentially that used by Fisher. The equation for laminar flow of a gas through a capillary tube, omitting corrections for slip, end effects, and expansion is

$$
F=\frac{\pi r^{4}}{16 l} \frac{\left(p_{1}^{2}-p_{2}^{2}\right)}{p \eta}
$$

where

$F=$ volume of gas discharged per unit time, measured at pressure $p$

$r=$ radius of tube

$l=$ length of tube

$p_{1}=$ entrance pressure

$p_{2}=$ exit pressure

$p=$ average of $p_{1}$ and $p_{2}$

$\eta=$ viscosity of the gas.

This may be rewritten

$$
\left(p_{1}^{2}-p_{2}^{2}\right)=\frac{16 p F \eta l}{\pi r^{4}} .
$$

The tube may be considered to be made up of elements in series, having slightly varying radii. For each elemental length, $d l$,

whence

$$
d\left(p^{2}\right)=\frac{16 p F \eta}{\pi} \frac{d l}{r^{4}},
$$

$$
\int d\left(p^{2}\right)=p_{1}^{2}-p_{2}^{2}=\frac{16 p F \eta}{\pi} \int_{l_{1}}^{l_{2}} \frac{d l}{r^{4}},
$$

where $d$ is the diameter of the capillary tube (inside).

Evaluation of the integral in the right-hand member is accomplished by measurement of the mercury thread. Neglecting, temporarily, the corrections due to the curvature of the ends, and assuming that we are dealing with a thread having flat ends, we let

$\lambda=$ length of the thread

$\eta=$ cross-sectional area of the tube

$v=$ volume of the thread

$\eta=\pi r^{2}=v / \lambda$

$\frac{l}{r^{4}}=\frac{\pi^{2} \lambda^{2}}{v^{2}}$.

Therefore,

$$
\int_{l_{1}}^{l_{2}} \frac{d l}{r^{4}}=\frac{\pi^{2}}{v^{2}} \int_{l_{1}}^{l_{2}} \lambda^{2} d l .
$$

If the observed values of $\lambda^{2}$ are plotted as a function of the position of the center of the thread from an arbitrary origin on the tube, the value of $\int_{l_{1}}^{l_{2}} \lambda^{2} d l$ may be found graphically. The evaluation of $v$ by direct weighing is difficult, because the sample is very small; in this case, averaging about $0.01 \mathrm{~g}$. The volume may be calculated from the weight of a long mercury thread by the following means.

The volume of an elemental section of the tube is related to the cross-sectional area of the tube by the equation

$$
d V=Q d l=\frac{v}{\lambda} d l .
$$

The volume, $V$, of any portion of the tube can be found by integrating between appropriate limits.

$$
V=v \int_{l_{3}}^{l_{4}} \frac{l}{\lambda} d l
$$

Solving this equation for the volume of the thread,

$$
v=\frac{V}{\int_{l_{3}}^{l_{4}} \frac{l}{\lambda} d l} .
$$

The volume, $V$, may be measured Iby means of a relatively long mercury thread, whose weight is great enough to be determined accurately by weighing. The integral is evaluated graphically. By substituting eq 7 in eq 5

$$
\int_{l_{1}}^{l_{2}} \frac{d l}{r^{4}}=\frac{\pi^{2}}{V^{2}} \int_{l_{1}}^{l_{2}} \lambda^{2} d l\left[\int_{l_{3}}^{l_{4}} \frac{l}{\lambda} d l\right]^{2}
$$

Combining eq 4 and 8 and solving for $\eta$ gives

$$
\eta=\frac{p_{1}^{2}-p_{2}^{2}}{p F} \frac{V^{2}}{16 \pi \int_{l_{1}}^{l_{2}} \lambda^{2} d l\left[\int_{l_{3}}^{l_{4}} \frac{l}{\lambda} d l\right]^{2}} .
$$

The right-hand fraction thus becomes a calibration factor associated with the tube, corresponding to the constant $\pi r^{4} / 16 l$ in eq 1 .

$$
K=\frac{V^{2}}{16 \pi \int_{l_{1}}^{l_{2}} \lambda^{2} d l\left[\int_{l_{3}}^{l_{4}} \frac{l}{\lambda} d l\right]^{2^{\prime}}}
$$

where $K$ is the flow constant. 
Corrections for curvature of meniscus. The photographs of the mercury threads, when examined under a magnification of 50 diameters, were found to have rounded menisci, which in most cases were nearly hemispherical. Several examples are shown in figure 3 , ranging from nearly perfect to badly distorted. As accurate measurement of the cylindrical portion of a thread would be difficult to make, it was decided to measure the over-all length and to reduce this observation to the length of a thread of the same total volume having flat ends. This involves measurement of the heights of the curved menisci and the difficulty of observing the point of contact of the mercury surface with the tube wall, but since the corrections are small, this measurement need not be made with great precision. Furthermore, it was found possible to draw a circle on the screen of the projection microscope, and to use this as a test for curvature of the menisci. Those which conformed closely to the circle could be assumed to be hemispherical and the meniscus height, equal to the average radius of the tube, used in computing the correction. About 80 percent of the photographs were so treated. In the remaining cases, the heights were measured and corrections computed by the following method.

In figure $4, h$ represents the observed meniscus height and $r$ the tube radius. The shaded portion represents a cylindrical element having the same volume as the spherical segment. The volume, $v$, of a segment of a sphere of height $h$ and radius of base $r$ is

$$
v=\frac{1}{6} \pi h\left(h^{2}+3 r^{2}\right) .
$$

This volume is equal to that of the cylindrical element, shaded in figure 4. As we wish to determine the change in length that the thread would undergo if it were altered from a curved to a flat end, we may denote the change of length by $S$, giving

$$
\pi r^{2}(h-S)=\frac{1}{6} \pi h\left(h^{2}+3 r^{2}\right) .
$$

Solving for $S$ gives

$$
S=\frac{h}{6}\left(3-\frac{h^{2}}{r^{2}}\right) .
$$

Corrections varied from about 0.0034 in. for a wellrounded meniscus such as shown at $\mathrm{A}$, figure 3 , to about 0.0012 in. for a badly flattened one. The correction for case $B$, figure 3 , was 0.0014 in.

Correction for target focus distance. Because of the finite distance of the X-ray source from the photographic plate, the image of the thread is slightly enlarged. The amount of this correction was minimized by keeping the tube in close contact with the paper envelope enclosing the photographic plate, and by having the distance from the plate to the X-ray target as great as permissible with reasonable exposure time. The greatest magnification factor encountered was 1.0016 , giving a maximum correction of about $0.0005 \mathrm{in}$.
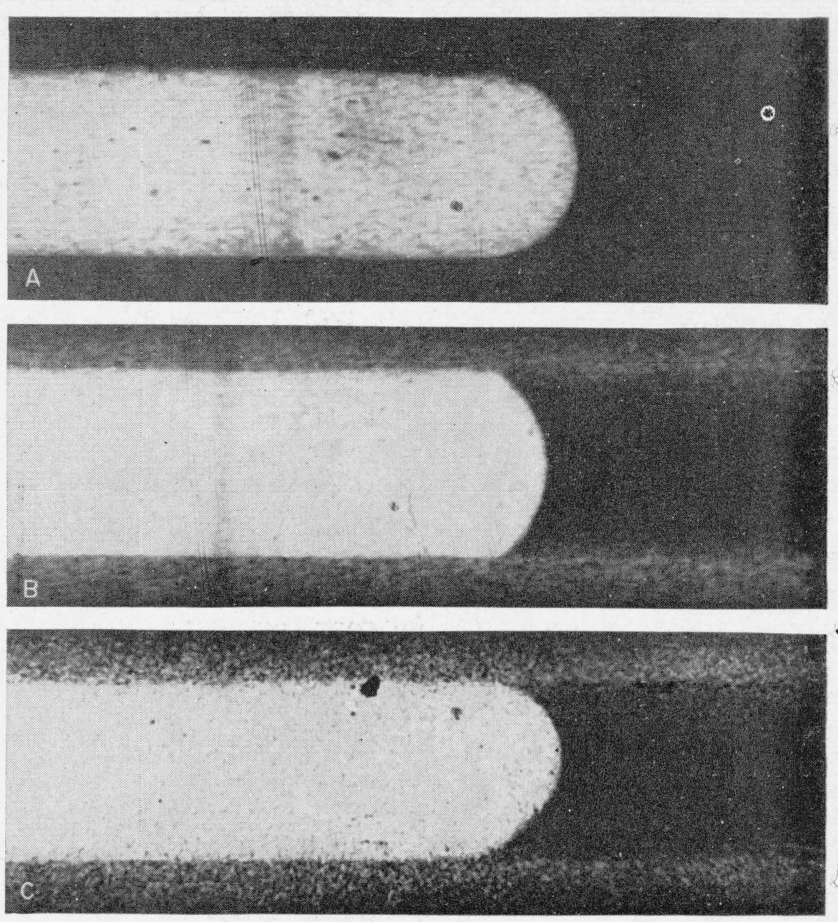

Figure 3. Examples of typical photographs.

$A$, Normal well-rounded meniscus; $B$, flattened meniscus; $C$, badly distorted meniscus.

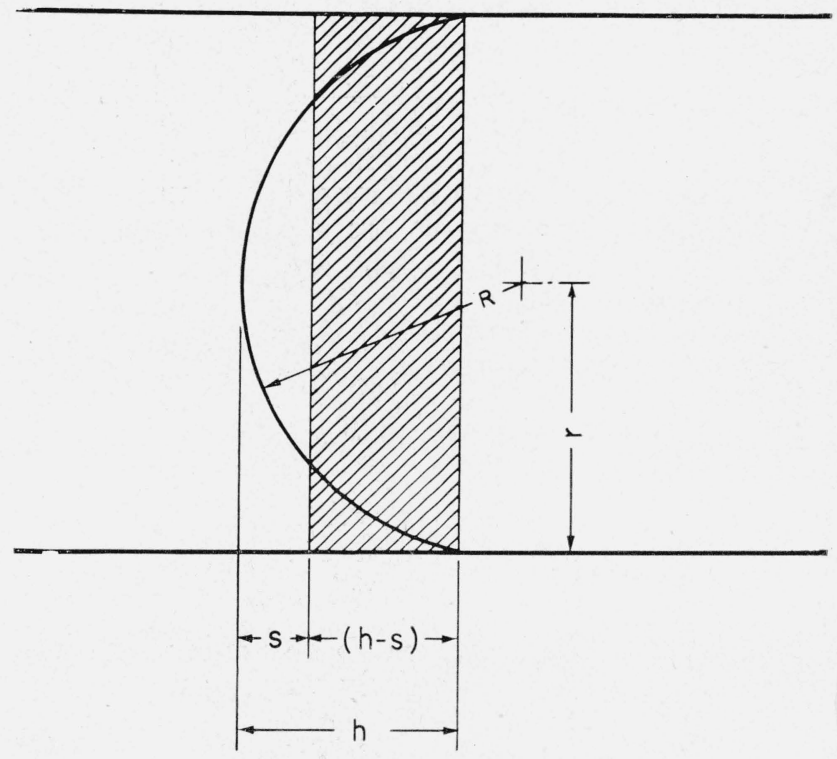

FIGURE 4. Illustrating method of correcting for curvature of meniscus.

\section{Results of Measurements}

Figure 5 illustrates portions of typical graphs of $\lambda^{2}$ and $1 / \lambda$ and the repeatability to be expected from consecutive trials with the same tube. Since the thread volume could not be duplicated exactly in the second trial, the plots are slightly displaced, but the general configuration correlates well.

Graphical integration was carried out in this case 

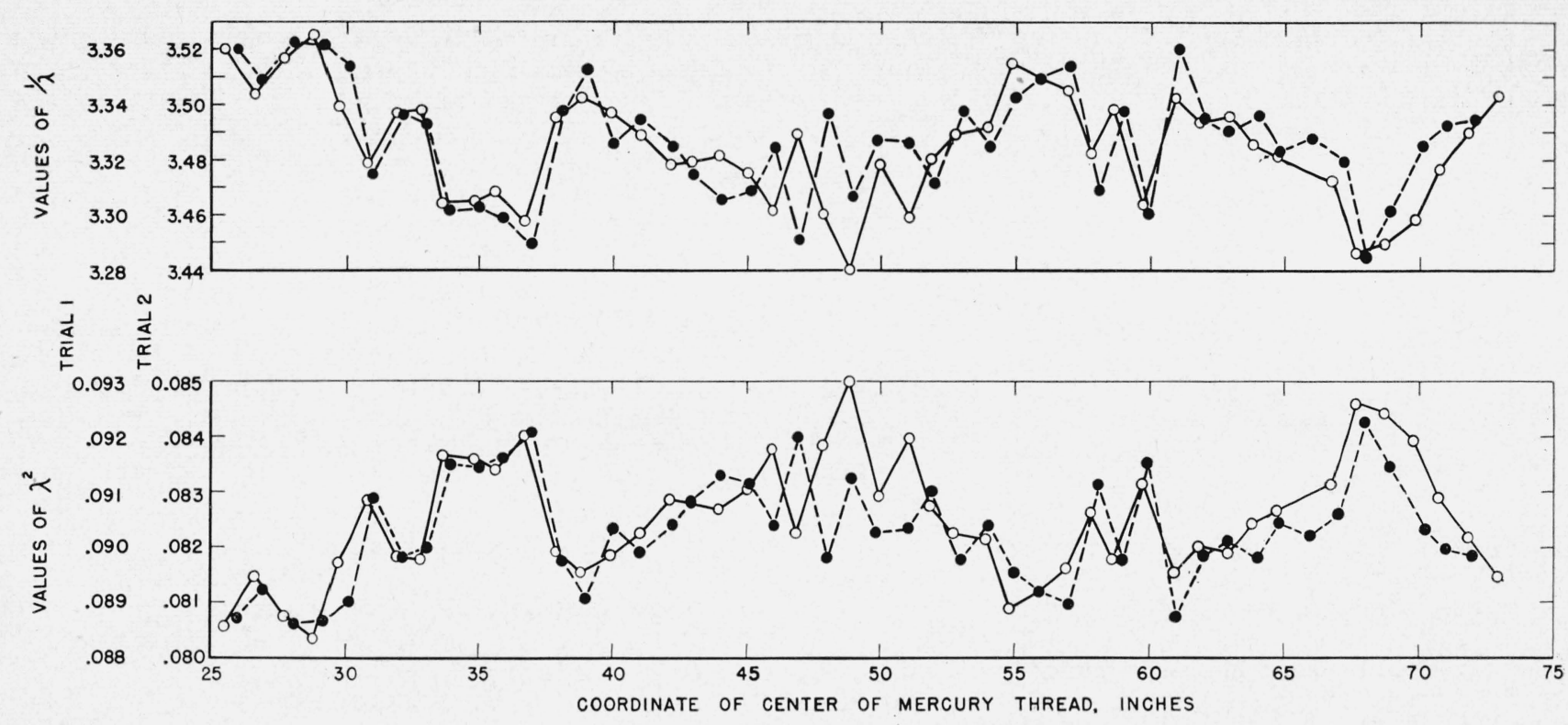

FIGURE 5. Typical graphs of $1 / \lambda$ and $\lambda^{2}$ used in graphical integration for calculating flow constants of capillary tubes.

Two independent trials of the same tube illustrating degree of repeatability.

by a weighing method. The plots were prepared on strips of graph paper approximately $6 \mathrm{ft}$. long. The width of the strip depended upon the variations encountered in the function, but was about 6 to 12 in. After plotting, the paper was cut along the pencil line and along the limits of integration. Each piece of paper was compactly folded and was weighed after conditioning overnight in the case of the balance, to achieve humidity equilibrium. The ratio of the weight of the lower section of the paper to the combined weight of the two parts was computed. The combined area calculated from the dimensions of the strip was multiplied by the ratio of the weights to give the area beneath the curve.

This method of graphical integration is subject to errors due to nonuniformity of the paper, differences in the amount of inking of the ruling, and unequal soiling of the paper surface in handling. In this instance, the area integrated between the curve and the edge of the strip of paper was only a small per- centage of the total area between the curve and the $x$-axis. In the integration of the $\lambda^{2}$ curves, the portion evaluated graphically was about 10 percent of the total area to the $x$-axis, whereas in the case of the $1 / \lambda$ integrals, it amounted to only about 3 percent. As a further check, the areas for tube 2 were computed by the trapezoidal rule and by Simpson's rule, and the results of both methods of computation agreed with that obtained by the weighing method within \pm 0.1 percent.

Values of the flow constant, $K$, are listed in column 2 of table 1 . For comparison, column 3 lists values of $\pi r^{4} / 16 l$, using for $r$ the average radius calculated from the weight of a long mercury thread.

Another method of illustrating the effect of irregularities in the tube is to compare the actual length of the tube with the length of an equivalent uniform tube whose radius is the same as that determined by the long mercury thread. These tube lengths are presented in columns 4 and 5 .

TABLE 1. Results of measurements of eight capillary tubes

\begin{tabular}{|c|c|c|c|c|c|c|c|c|}
\hline Tube number & Flow constant, & $\pi r^{4} / 16 l$ & $\begin{array}{c}\text { Length of } \\
\text { tube }\end{array}$ & $\begin{array}{c}\text { Length of } \\
\text { equivalent } \\
\text { tube of } \\
\text { uniform } \\
\text { bore }^{\text {a }}\end{array}$ & $\begin{array}{l}\text { Average } \\
\text { inside } \\
\text { diameter }\end{array}$ & $\begin{array}{l}\text { Maximum } \\
\text { plus de- } \\
\text { parture } \\
\text { from } \\
\text { average } \\
\text { diameter }\end{array}$ & $\begin{array}{l}\text { Maximum } \\
\text { minus de- } \\
\text { parture } \\
\text { from } \\
\text { average } \\
\text { diameter }\end{array}$ & $\begin{array}{l}\text { Average } \\
\text { departure } \\
\text { from } \\
\text { average } \\
\text { diameter }\end{array}$ \\
\hline 1 & 2 & 3 & 4 & 5 & 6 & 7 & 8 & 9 \\
\hline $\begin{array}{l}1 \\
1 \\
1 \\
2 \\
1 \\
1 \\
1 \\
1\end{array}$ & $\begin{array}{l}\text { 3. } 375 \times 10^{-12} \\
\text { 3. } 380 \\
\text { 3. } 278 \\
\text { 2. } 954 \\
\text { 3. } 660 \\
\text { 3. } 782 \\
\text { 3. } 646 \\
\text { 3. } 609 \\
\text { 3. } 751\end{array}$ & $\begin{array}{l}\text { 3. } 389 \times 10^{-12} \\
\text { 3. } 389 \\
\text { 3. } 282 \\
\text { 2. } 961 \\
\text { 3. } 658 \\
\text { 3. } 793 \\
\text { 3. } 657 \\
\text { 3. } 609 \\
\text { 3. } 753\end{array}$ & $\begin{array}{l}\text { in. } \\
160.00 \\
160.00 \\
165.29 \\
168.00 \\
172.69 \\
174.00 \\
174.00 \\
174.00 \\
172.00\end{array}$ & $\begin{array}{l}160.66 \\
160.43 \\
165.49 \\
168.39 \\
172.60 \\
174.50 \\
174.52 \\
174.00 \\
172.09\end{array}$ & $\begin{array}{l}\text { in. } \\
0.01450 \\
.01450 \\
.01450 \\
.01490 \\
.01506 \\
.01523 \\
.01509 \\
.01504 \\
.01514\end{array}$ & $\begin{array}{r}\text { Percent } \\
1.58 \\
1.46 \\
.98 \\
1.13 \\
1.49 \\
2.45 \\
1.24 \\
1.08 \\
1.20\end{array}$ & $\begin{array}{r}\text { Percent } \\
0.93 \\
1.11 \\
1.28 \\
.45 \\
1.10 \\
1.06 \\
1.08 \\
1.66 \\
1.40\end{array}$ & $\begin{array}{r}\text { Percent } \\
0.47 \\
.43 \\
.29 \\
.21 \\
.35 \\
.44 \\
.36 \\
.39 \\
.34\end{array}$ \\
\hline
\end{tabular}

a The values presented in these columns are subject to an uncertainty of approximately \pm 0.2 percent.

$904428-50-3$ 
For any position of the short mercury thread, the diameter is inversely proportional to the square root of the thread length.

$$
\frac{d_{1}}{d_{0}}=\sqrt{\frac{\lambda_{0}}{\lambda_{1}}}
$$

where $d_{0}$ is the average diameter of the capillary tube.

We may compute the percentage deviation of the diameter at any point from the average diameter from the equation

$$
D=100\left(\sqrt{\frac{\lambda_{0}}{\lambda}}-1\right)
$$

in which

$$
\begin{aligned}
D & =\text { the percentage departure. } \\
\lambda & =\text { the observed thread length. } \\
\lambda_{0} & =\text { the average of the observed thread lengths. }
\end{aligned}
$$

Columns 7 and 8 of table 1 list the maximum positive and negative departures from the average diameter, and column 9 lists the average departure of the individual determinations from the average diameter.

The tubes studied were not all made by the same manufacturing process. The starting material in each case was tubing having an outside diameter of approximately $1 \frac{1}{4}$ in. Tubes 1 to 3 were drawn over steel mandrels through dies that reduced the diameter by about one-quarter at each step, until the inside diameter was about 0.09 in. Thereafter the reductions were accomplished without mandrels.

A second set of tubes (not listed in table 1) were obtained from a manufacturer who drew the tubes to the finish diameter over mandrels. It was found that the rolls used in loosening the tubing from the mandrels were not perfectly centered and therefore produced a periodic variation in the diameters, which rendered the tubes unuseable. The variations amounted to as much as \pm 5 percent. Attempts were made to eliminate these differences by successive drawing through external dies, with slight diameter reduction. After four stages, the variations were somewhat reduced, but the periodic character persisted and the tubes were abandoned. Tubes 8 to 12 , with heavier walls, were then supplied by the same manufacturer. In a lot of six tubes, only one was found to have unsatisfactory characteristics.

\section{Summary}

It has been found practical to apply the method of Fisher to the determination of flow constants for metallic capillary tubing and to study the uniformity of such tubing by X-ray photography of a mercury thread within the tube. Eight pieces of tubing were selected from a group of twelve, having a maximum deviation from the average diameter of 2.5 percent. Flow constants for these eight tubes were determined.

Washington, April 18, 1950.

\title{
Mass Spectra of Some Simple Isotopic Molecules
}

\author{
By Vernon H. Dibeler, Fred L. Mohler, Edmund J. Wells, Jr., and Robert M. Reese \\ The mass spectra of $\mathrm{H}_{2}, \mathrm{D}_{2}, \mathrm{~T}_{2}, \mathrm{HD}$, and $\mathrm{HT}$ have been measured with a Consolidated \\ mass spectrometer with 50 -volts ionizing voltage and with a constant magnetic field. The \\ following ratios of atom ions to molecule ions were observed: $\mathrm{H}^{+} / \mathrm{H}_{2}^{+}=0.0201, \mathrm{D}^{+} / \mathrm{D}_{2}^{+}=$ \\ $0.0096, \quad \mathrm{~T}^{+} / \mathrm{T}_{2}^{+}=0.0061, \quad \mathrm{H}^{+} / \mathrm{HD}^{+}=0.0096, \quad \mathrm{D}^{+} / \mathrm{HD}^{+}=0.0097, \quad \mathrm{H}^{+} / \mathrm{HT}^{+}=0.0085, \quad$ and \\ $\mathrm{T}^{+} / \mathrm{HT}^{+}=0.0068$. The ratios for $\mathrm{T}_{2}$ and $\mathrm{HT}$ were derived from spectra of two samples \\ containing 91 and 37 atomic percent of $\mathrm{T}$ in equilibrium with $\mathrm{H}$. Theoretical values for the \\ ratios were computed for ionization involving transition into the $\Sigma_{g}$ state of the molecule ion. \\ These values are lower than the observed ratios, because transitions to higher repulsive \\ states contribute to the observed values at 50 -volts ionizing voltage. \\ Mass spectra of $\mathrm{CO}$ and $\mathrm{CO}_{2}$ containing about 54 atom percent $\mathrm{C}^{13}$ were measured \\ under standard operating conditions. For $\mathrm{CO}$ the ratio $\mathrm{C}^{12+} / \mathrm{C}^{12} \mathrm{O}^{+}=0.0540$ and $\mathrm{C}^{13+} /-$ \\ $\mathrm{C}^{13} \mathrm{O}^{+}=0.0514$. For $\mathrm{CO}_{2}$, the ratios are $\mathrm{C}^{12} \mathrm{O}^{+} / \mathrm{C}^{12} \mathrm{O}_{2}^{+}=0.0868, \mathrm{C}^{13} \mathrm{O}^{+} / \mathrm{C}^{13} \mathrm{O}_{2}^{+}=0.0824$, \\ $\mathrm{C}^{12+} / \mathrm{C}^{12} \mathrm{O}^{+}=0.0564$, and $\mathrm{C}^{13}+/ \mathrm{C}^{13} \mathrm{O}_{2}^{+}=0.0542$. \\ A theory of the isotope effect in $\mathrm{CO}$ involving simplifying assumptions indicates that \\ the relative probability of producing $\mathrm{C}^{12}+$ is 3.5 percent greater than the probability of pro- \\ ducing $\mathrm{C}^{13+}$, as compared with an observed difference of 5 percent.
}

\section{Isotopic Hydrogen Molecules}

\section{Introduction}

Mass spectra of $\mathrm{H}_{2}$ and $\mathrm{D}_{2}$ have been investigated frequently [1], ${ }^{1}$ and a recent paper by Friedel and

${ }_{1}$ Figures in brackets indicate the literature references at the end of this paper.
Sharkey [2] gives the spectrum of HD. The present research was undertaken because of the opportunity to obtain some very pure HD and a sample of tritium of relatively good purity. Hydrogenic spectra are of interest because it is possible to compute theoretically from the Franck-Condon principle the relative probabilities of producing atom ions from the differ- 